\title{
ON THE TAUTNESS PROPERTY OF ALEXANDER-SPANIER COHOMOLOGY
}

\author{
SATYA DEO
}

ABSTRACT. Any neighborhood retract of an arbitrary topological space is tautly imbedded with respect to Alexander-Spanier cohomology.

Introduction. It has been remarked [2, p. 337] that a point subspace of any topological space is tautly imbedded with respect to Alexander-Spanier cohomology. The objective of this paper is to show that this observation can be generalized in the sense that every neighborhood retract, in particular every retract of any space, is a taut subspace with respect to AlexanderSpanier cohomology. In order to indicate the sharp contrast between the Alexander-Spanier cohomology and the singular cohomology with respect to tautness property, we include an example of a compact Hausdorff space such that none of its points is taut with respect to the latter. An application of our result is also given.

All definitions and notations used follow those in [2]. Our main result is the following

Theorem. Let $A$ be any subspace of a topological space $X$ which is a neighborhood retract of $X$. Then $A$ is a taut subspace of $X$ with respect to Alexander-Spanier cohomol:gy, i.e., for every $p \geq 0$ and every coefficient module $G$, there is an isomorphism $\lim _{\longrightarrow} \bar{H}^{p}(U, G) \simeq \bar{H}^{p}(A, G)$ where $U$ runs over all the open neighborhoods of $A$ in $X$.

Proof. Let $M$ be an open neighborhood of $A$ in $X$ and $r: M \rightarrow A$ be a retraction. Obviously, it suffices to consider only those open $U$ of $X$ which are contained in $M$. Let $\eta: \lim _{\longrightarrow} \bar{H}^{p}(U, G) \rightarrow \bar{H}^{p}(A, G)$ denote the homomorphism induced by the restriction homomorphisms $i^{*}: \bar{H}^{p}(U, G) \rightarrow \bar{H}^{p}(A, G)$ where $i: A \rightarrow U$ is the inclusion map. Since $A$ is a retract of each of the open sets $U, \eta$ is an epimorphism.

To prove that $\eta$ is also a monomorphism, let $\{\bar{\alpha}\} \in \bar{H}^{p}(U, G)$, where $a \in C^{p}(U, G)$ and $\bar{\alpha}$ denotes the coset of $a$ with respect to $C_{0}^{p}(U, G)$, and $\{\bar{\alpha}\}$ is the cohomology class of the cocyle $\bar{\alpha}$. Let $U^{\prime}=\left\{U^{\prime}\right\}$ be an open covering of $U$ in $X$ such that $\delta \alpha=0$ on $U^{\prime p+2}$. Now suppose that $\{\bar{\alpha}\} \mid A=0 \epsilon$ $\bar{H}^{p}(A, G)$. Then we have an open covering $U^{\prime \prime}=\left\{U^{\prime \prime}\right\}$ of $A$ in $X$ and an $\alpha^{\prime} \epsilon$ $C^{p-1}(A, G)$ such that $\delta \alpha^{\prime}=\alpha \mid A$ on $U^{\prime \prime p+1}$. We need to show only that there

Received by the editors July 3, 1973. $55 \mathrm{~B} 10$.

AMS (MOS) subject classifications (1970). Primary 55B05, 55B99; Secondary 
is an open set $N$ of $X$ such that $A \subset N \subset U$ and $\{\bar{a}\} \mid N=0 \in \bar{H}^{p}(N, G)$. Define

$$
\mathcal{W}=\left\{W \mid W=U^{\prime} \cap U^{\prime \prime}, U^{\prime} \in \mathcal{U}^{\prime}, U^{\prime \prime} \in \mathcal{U}^{\prime \prime} \text { and } U^{\prime} \cap A \neq \varnothing \neq U^{\prime \prime} \cap A\right\}
$$

and

$$
\mathcal{O}=\left\{V \mid V=W \cap r^{-1}(W \cap A), W \in\langle i\}\right.
$$

where $r=r \mid U: U \rightarrow A$ is the retraction map. Notice that $\mathcal{O}$ is an open covering of $A$ in $X$ and that for every $x \in V \in \mathcal{O}, r(x) \in V$. Now put $N=\bigcup\{V \mid V$ $\epsilon$ O\}. Then again if $r=r \mid N: N \rightarrow A$ is the retraction map we have

$$
\delta r^{\sharp}\left(\alpha^{\prime}\right)=r^{\#}\left(\delta \alpha^{\prime}\right)=r^{\sharp}(\alpha \mid A) \text { on } \bigcirc^{p+1} \text {. }
$$

Hence it only remains to prove that $\{\bar{\alpha}\} \mid N=\left\{\bar{r}^{\sharp}(\alpha \mid A)\right\} \in \bar{H}^{p}(N, G)$. For that let us define $D \phi \in C^{p-1}(N, G)$ for $\phi \in C^{p}(N, G)$ by

$$
(D \phi)\left(x_{0}, \cdots, x_{p-1}\right)=\sum_{0 \leq i \leq p-1}(-1)^{i} \phi\left(x_{0}, \ldots, x_{i}, r\left(x_{i}\right), \ldots, r\left(x_{p-1}\right)\right) .
$$

Then it is not hard to verify that $\delta D \phi+D \delta \phi=r^{\sharp}(\phi \mid A)-\phi$. Since $x \in V$ implies $x, r(x) \in V \subset U^{\prime}$ for some $U^{\prime} \in \mathcal{U}^{\prime}$, we have

$$
\delta(\alpha \mid N)\left(x_{0}, \cdots, x_{p+1}\right)=0 \text { on } \oslash^{p+2} .
$$

Whence

$$
\delta D(\alpha \mid N)=r^{\sharp}(\alpha \mid A)-\alpha \mid N \text { on } \ell^{p+1}
$$

Now passing to the cocycles we have the desired result.

Corollary 1. Every retract of an arbitrary topological space is a taut sub. space of $X$ with respect to Alexander-Spanier cohomology.

Corollary 2. Let $(A, B)$ be a pair of subspaces of a space $X$ such that each of them is a neighborhood retract in $X$. Then the pair $(A, B)$ is taut with respect to Alexander-Spanier cohomology.

Remark 1. The technique of the proof is essentially the same as used by Spanier [2, p. 316] in proving that a closed subset of a paracompact Hausdorff space is taut with respect to Alexander-Spanier cohomology. Moreover, a close examination would indicate that in the proof of both of these results the fundamental lemma of Spanier [3] with respect to the maps $I_{N}$ and $r \mid N: N \rightarrow A$ has been used.

Remark 2. Since the Čech cohomology and the Alexander-Spanier cohomology are naturally isomorphic on the category of all topological pairs [1], all of our results are valid for Čech cohomology also. However, it is not true for singular cohomology because even the point-subspaces need not be taut in spaces beyond the class of homologically locally connected ones as the following example shows. 
Example. Let $X=\Pi_{i \in I} S_{i}^{m}$ be an infinite product of $m$-spheres, $m>1$. For $m=1$ it can also be modified without any important change. Let $x=$ $\left(x_{i}\right) \in X$ and notice that any neighborhood $U(x)$ of $x$ in $X$ is of the same homotopy type as $X$ itself. Of course the inclusion map $i: U(x) \rightarrow X$ need not be a homotopy equivalence. By suitable excision it can be seen that

$$
H_{q}(X, U(x)) \simeq \begin{cases}\bigoplus_{i \in F} Z_{i}, & q=m, \\ 0, & \text { otherwise },\end{cases}
$$

where $H_{q}$ denotes the reduced integral singular homology groups and $Z_{i}=Z$ is the additive group of integers for each $i$ and $F$ is some finite subset of $I$. The following sequence, therefore, is exact:

$$
0 \rightarrow H_{m}(U(x)) \stackrel{i_{*}}{\longrightarrow} H_{m}(X) \rightarrow H_{m}(X, U(x)) \rightarrow 0 .
$$

Recall [2, Example 7B5] that $\pi_{n}\left(\Pi S_{i}^{m}\left(x_{i}\right)\right) \simeq \Pi\left(\pi_{n}\left(S_{i}^{m}, x_{i}\right)\right)$ and that the $n$. sphere is $(n-1)$-connected. Hence, by the Hurewicz theorem [2, Theorem 7.5.5], $H_{m}(X) \simeq \Pi_{i \in I} Z_{i}$. Since $H_{m-1}(X)=0$, we have, by the mixed homology cohomology theorem for singular theory, $H^{m}(X, Q) \simeq \operatorname{Hom}\left(\Pi_{i \in I} Z_{i}, Q\right)$, where $Q$ is the additive group of rationals. For each $U(x)$ we have a commutative diagram

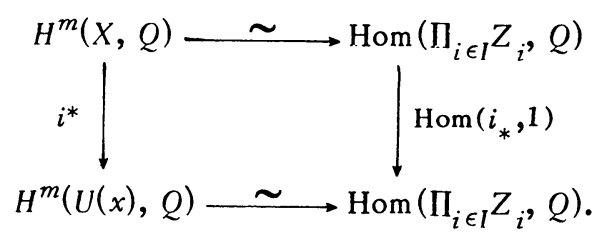

Now let $f: \bigoplus Z_{i} \rightarrow Q$ be the homomorphism such that $f\left(1_{i}\right)=1$ for each generator $1_{i}$ of $Z_{i}$. Injectivity of $Q$ guarantees a homomorphism $\bar{f}: \Pi Z_{i} \rightarrow Q$ extending $f$. If $\bar{c} \in H^{m}(X, Q)$ corresponds to $f$ under the above isomorphism, then $\bar{c} \mid U(x) \neq 0$ for each neighborhood $U(x)$ of $x$ in $X$. This completes the proof of our assertion.

Now the following interesting application of our result implies that in the homotopy axiom of Alexander-Spanier cohomology, and hence Čech cohomology for the compact spaces, the unit interval can be replaced by any connected space $T$.

Corollary 3. Let $T$ and $X$ be any connected and compact spaces, respectively, and $h_{t}: X \rightarrow X \times T$ the family of maps defined by $h_{t}(x)=(x, t)$. Then $h_{t}^{*}: \bar{H}^{*}(X \times T, G) \rightarrow \bar{H}^{*}(X, G)$ is independent of $t$.

Proof. It suffices to prove that if $c \in \bar{H}^{*}(X \times T, G)$ is such that $c \mid X \times$ $t=0$ for any $t \in T$ then there is a neighborhood $U(t)$ of $t$ in $T$ such that $c \mid X \times t^{\prime}=0$ for every $t^{\prime} \in U(t)$. But that follows at once since $X \times t$ being a 
retract of $X \times T$ is tautly imbedded and $X$ is compact.

The author is thankful to Professor John W. Keesee for useful discussions during the preparation of this paper.

\section{REFERENCES}

1. C. H. Dowker, Homology groups of relations, Ann. of Math. (2) 56 (1952), 84-95. MR 13, 967.

2. E. H. Spanier, Algebraic topology, McGraw-Hill, New York, 1966. MR 25 $\# 1007$.

3. - Cohomology theory for general spaces, Ann. of Math. (2) 49 (1948), 407-427. MR 9, 523.

DEPARTMENT OF MATHEMATICS, UNIVERSITY OF ARKANSAS, FAYETTEVILLE, ARKANSAS 72701

Current address: Department of Mathematics, Allahabad University, Allahabad211002, India 